\title{
Antenna Independent Path Loss Model for In-Body Communication in Homogeneous Tissue
}

\author{
Divya Kurup, Wout Joseph, Emmeric Tanghe, Günter Vermeeren, and Luc Martens \\ Wireless and Cable (WiCa) Group, IBBT/INTEC, Ghent University \\ Gaston Crommenlaan 8 box 201, B-9050 Ghent, Belgium \\ Fax: +32 933 14899, E-mail: divya.kurup@ intec.UGent.be
}

\begin{abstract}
In this paper we propose for the first time an inbody path loss model for homogeneous human muscle tissue that is independent of the antennas for in-body communication at 2.45 GHz. The path loss model obtained can be used to design in-body communication systems at $2.45 \mathrm{GHz}$.
\end{abstract}

\section{INTRODUCTION}

A wireless body area network (WBAN) consists of nodes that communicate wirelessly and are located on or in the body of a person. A good understanding of propagation loss within human body is required for the development of WBAN for implants within human body. The human body is a lossy medium hence a considerable attenuation of the waves traveling from the transmitter $(\mathrm{Tx})$ to the receiver $(\mathrm{Rx})$ takes place. By understanding the path loss (PL) within human body optimum communication can be established between the nodes placed within the body. In literature various PL models have been proposed for in-body propagation but they do not extract the gain of the antenna and are thus antenna dependent [1]. In this paper, we develop an in-body PL independent of the antenna by extracting the antenna gains from the PL for two different types of antennas within homogeneous lossy human muscle tissue.

\section{METHOD}

Wave propagation is investigated in human muscle tissue (relative permittivity $\epsilon_{r}=50.8$ and conductivity $\sigma=2.01$ $\mathrm{S} / \mathrm{m}$ [2]) using two pairs of insulated antennas at $2.45 \mathrm{GHz}$. Insulated antennas are selected instead of bare antennas because the insulated antenna prevents the leakage of conducting charges from the antenna and also reduces the sensitivity of the entire distribution of current to the electrical properties of the ambient medium. This property makes insulated antennas valuable for in-body communication [3].

The first pair of antennas consist of two identical insulated dipoles where the dipole arms are perfect electric conductors (PEC) surrounded by an insulation made of polytetrafluoroethylene (PTFE) $\left(\epsilon_{r}=2.07\right.$ and $\left.\sigma=0 \mathrm{~S} / \mathrm{m}\right)$. The length of the dipole arms is chosen to be $\ell_{1}=3.9 \mathrm{~cm}$ (henceforth addressed as antenna $a_{(\lambda / 2)}$ ) such that the antenna resonates at $2.45 \mathrm{GHz}$. The resonance appears when the antenna is equal to half the wavelength in a homogeneous medium equivalent to the combination of the insulation and the muscle tissue medium. Hence, $\lambda_{\text {res }}=7.8 \mathrm{~cm}$ (where, $\lambda_{\text {res }}$ is the wavelength at which resonance occurs) and we can derive the equivalent permittivity $\epsilon_{r, \text { equiv }}=2.45$ which is closer to the permittivity of the insulation.

The second pair of antennas are also dipole antennas insulated with the same insulation of PTFE. The length of the dipole arms $\ell_{2}=7.8 \mathrm{~cm}$ (henceforth addressed as $\left.\operatorname{antenna}_{(\lambda)}\right)$.

\section{A. Simulation}

Simulations are carried out for the setup of the insulated antennas in human muscle tissue medium for a separation of $30 \mathrm{~cm}$ between the $\mathrm{Tx}$ and the Rx using FEKO, a method of moments (MoM) program. For accurate modelling in the MoM tool, segmentation rules are adhered (segment length $=\lambda_{\text {res }} / 12$, edge length $=\lambda_{\text {res }} / 12$ ). The source used is a current source.

\section{RESULTS}

\section{A. Gain of the antenna in conductive medium}

The gain of the antenna in free space is defined as the ratio of radiation intensity in a particular direction to the radiation intensity of an isotropic antenna [4]. However, the radiation intensity becomes distance dependent in a conducting medium. To make the antenna gain distance independent in a conducting medium, it can be expressed as follows [4]:

$$
G=\left(4 \pi R g^{2}\right) / R_{r}
$$

where, $G$ is the gain of the antenna in the conductive medium and $g$ is a function involving the parameters of the medium, $R$ is the intrinsic resistance and is equal to $\sqrt{\omega \mu / 2 \sigma}$ and $R_{r}$ is the radiation resistance. Also, $\omega=2 \cdot \pi \cdot f=$ angular frequency $[\mathrm{rad} / \mathrm{sec}], f=$ frequency $=2.45 \mathrm{GHz}, \mu=$ permeability of the lossy medium, and $\sigma=$ conductivity of the lossy medium $[\mathrm{S} / \mathrm{m}]$.

Further, $g$ is defined [4] as follows:

$$
g=\left(|H| d e^{d / \delta}\right) / I_{i}
$$

where, $|H|$ is the magnitude of the magnetic field in $[\mathrm{A} / \mathrm{m}]$ taken in the maximum field direction of the antenna under consideration at distance $d, \delta$ is the skin depth in [m], $I_{i}$ is the input current in $[\mathrm{A}]$ and $d$ is the distance in [m]. 


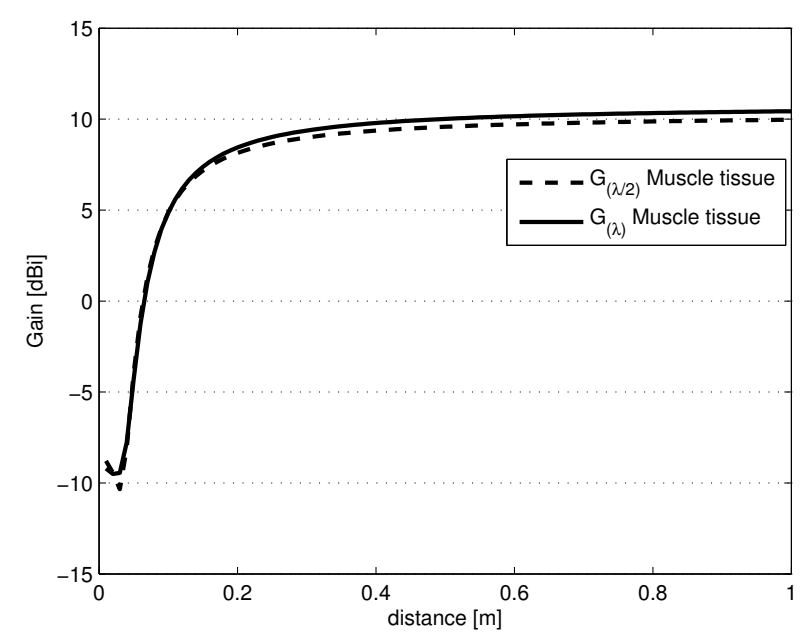

Fig. 1. Gain of the insulated dipole antennas in muscle tissue.

1) Gain of antenna $(\lambda / 2)$ and antenna $a_{(\lambda)}$ in muscle tissue: The gain of the antenna $a_{(\lambda / 2)}$ and antenna $a_{(\lambda)}$, henceforth referred to as $G_{(\lambda / 2)}$ and $G_{(\lambda)}$ respectively, in the muscle tissue is obtained using (1) and is shown in Fig. 1. $G_{(\lambda)}$ and $G_{(\lambda / 2)}$ are $10.7 \mathrm{dBi}$ and $8.7 \mathrm{dBi}$ in the muscle tissue, repectively. The values of the gain selected are the asymptotic values obtained at large distance where it is more or less constant. Fig. 1 shows that $G_{(\lambda / 2)}$ and $G_{(\lambda)}$ are independent of distance after $80 \mathrm{~cm} .|H|$ is obtained from the simulations, $I_{i}=1 \mathrm{~A}, \delta$ is $7.2 \mathrm{~mm}$.

\section{B. Path Loss}

PL between a pair of antennas is the ratio of transmitted power to the received power in decibels [5]:

$$
\left.P L\right|_{d B}=10 \cdot \log \left(P_{T} G_{T} G_{R} / P_{R} L_{T} L_{R}\right),
$$

where $P_{T}=$ transmit power,$P_{R}=$ received power, $G_{T}=$ transmitter antenna gain, $G_{R}=$ receiver antenna gain, $L_{T}=$ feeder loss at transmitter, $L_{R}=$ feeder loss at receiver. (3) can be written as follows when $L_{T}=L_{R}=0$ :

$$
\left.P L_{e x c l}\right|_{\mathrm{dB}}=-\left|S_{21}\right|_{d B}+\left.G_{T}\right|_{d B i}+\left.G_{R}\right|_{d B i},
$$

where, $\left|S_{21}\right|_{d B}$ is the forward transmission coefficient. Current literature [1] defines the in-body path loss $\left(P L_{i n c l}\right)$, which includes the gain and is thus antenna dependent, as $1 /\left|S_{21}\right|^{2}$ with respect to $50 \Omega$ when the generator at the Tx has an output impedance of $50 \Omega$ and the $\mathrm{Rx}$ is terminated with $50 \Omega$, this allows to regard the setup as a two-port circuit for which we determine $\left|S_{21}\right|_{\mathrm{dB}}$ with reference impedances of $50 \Omega$ at both ports. $P L_{\text {incl }}$ is defined as follows:

$$
\left.P L_{\text {incl }}\right|_{\mathrm{dB}}=\left(P_{T} / P_{R}\right)=-10 \log _{10}\left|S_{21}\right|^{2}=-\left|S_{21}\right|_{\mathrm{dB}},
$$

where, $P_{T}=$ input power at port 1 and $P_{R}=$ power received at port 2 in a two-port setup.

$P L_{\text {excl }}$ is now defined as the actual PL, i.e. excluding the gains and thus antennas independent [5]. The Tx and the Rx here are identical (Section II) hence the gain of the Tx and $\mathrm{Rx}$ are the same. $P L_{e x c l}$ is then calculated as follows from (4):

$$
\left.P L_{\text {excl }}\right|_{\mathrm{dB}}=\left.P L_{i n c l}\right|_{\mathrm{dB}}+2 G_{\mathrm{dBi}},
$$

where $G$ is the gain of the Tx and $\mathrm{Rx}$ antenna in $\mathrm{dBi}$ in the conductive medium according to (1).

Fig. 2 shows clearly that the $P L_{e x c l}$ for both the antennas agree well thus showing that an antenna independent PL model can be developed by determining the gain of the antenna. The gain obtained at larger separation i.e., $26 \mathrm{~cm}$ (Fig. 1, Section. III-A) is excluded from the path loss using (6). By excluding the gain, PL becomes antenna independent: mean deviation between $P L_{e x c l}$ of antenna $(\lambda)$ and $P L_{e x c l}$ of antenna $_{(\lambda / 2)}$ is only $1.53 \mathrm{~dB}$. Figs. 2 clearly shows that $P L_{i n c l}$ as used previously in literature, is antenna dependent with deviations up to $7-8 \mathrm{~dB}$ in the muscle tissue. The deviation in $P L_{e x c l}$ between antenna $(\lambda / 2)$ and antenna $(\lambda)$ exists only up to a certain distance (around $26 \mathrm{~cm}$ ) after which the PL merges because of the gain application at larger distance.We show here that using (1) and (6) we are able to define PL as it should be, namely antenna independent.

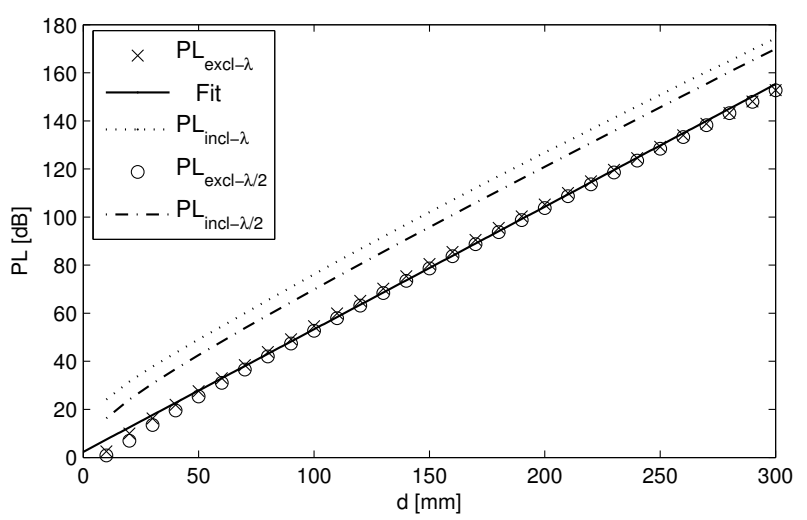

Fig. 2. PL of the insulated dipole including and excluding the gain in muscle tissue.

\section{Model for $P L$}

In this section, we propose a generalized model based on the simulation results for both the antennas in muscle tissue. The PL model as a function of distance in human muscle at $2.45 \mathrm{GHz}$ is as follows:

$$
\left.\left.P L_{\text {excl }}\right|_{\mathrm{dB}} \equiv P L\right|_{\mathrm{dB}}=\left(10 \log _{10} e^{2}\right) \alpha d+\left.C\right|_{\mathrm{dB}}
$$

where the parameters $\alpha$ is the attenuation constant $\left[\frac{1}{c m}\right],\left.C\right|_{\mathrm{dB}}$ is the constant, and $d$ is in $\mathrm{cm}$. $\left(10 \log _{10} e^{2}\right)$ equals $8.68 \mathrm{~dB}$ and shows the exponential behaviour of the PL. The parameter values for antenna independent PL are $\alpha=0.58\left[\frac{1}{\mathrm{~cm}}\right]$ and $\left.C\right|_{\mathrm{dB}}=1$ and are obtained by using a least square-error method. The attenuation constant obtained for the muscle tissue agrees well with the attenuation constant for plane wave which is $0.52\left[\frac{1}{\mathrm{~cm}}\right]$. In Figs 2 the $P L_{\text {excl }}$ in muscle tissue (for 
$\operatorname{antenna}_{(\lambda / 2)}$ and antenna $(\lambda)$ are fitted to the PL model of (7) with a mean deviation lower than $1.5 \mathrm{~dB}$. Thus, the proposed PL model is an excellent fit and can be used for any antenna in link budget calculations by introducing the antenna gain in tissue.

\section{Conclusions}

The path loss between different types of insulated dipole antennas is investigated at $2.45 \mathrm{GHz}$ in homogeneous human muscle tissue and for the first time an in-body path loss model independent of the antennas is derived by excluding the gain of the antennas. The PL model can thus be used to design any in-body communication system in muscle tissue and head tissue.

\section{REFERENCES}

[1] A. Alomainy and Y. Hao, "Modeling and characterization of biotelemetric radio channel from ingested implants considering organ contents," Antennas and Propagation, IEEE Transactions, vol. 57, pp. 999-1005, April 2009.

[2] FCC OET Bulletin 65, Revised Supplement C, "Evaluating Compliance with FCC Guidelines for Human Exposure to Radiofrequency Electromagnetic Fields," Federal Communication Commission, Office of Engineering and Technology, June 2001.

[3] R. W. P. King, G. S. Smith, M. Owens, and T. T. Wu, Antennas in matter fundamentals, theory and applications. Cambridge, MA : MIT Press, 1981.

[4] R. Moore, "Effects of a surrounding conducting medium on antenna analysis," Antennas and Propagation, IEEE Transactions, vol. 11, pp. 216-225, May 1963.

[5] S. R. Saunders, Antennas and Propagation for Wireless Communication Systems. Wiley and Sons, 1999. 